\title{
Dense dwarfs versus gelatinous giants: The trade-offs and physiological limits determining the body plan of planktonic filter feeders
}

\author{
Dölger, Julia; Kiørboe, Thomas; Andersen, Anders
}

\section{Published in:}

American Naturalist

Link to article, DOI:

$10.1086 / 703656$

Publication date:

2019

Document Version

Publisher's PDF, also known as Version of record

Link back to DTU Orbit

Citation (APA):

Dölger, J., Kiørboe, T., \& Andersen, A. (2019). Dense dwarfs versus gelatinous giants: The trade-offs and physiological limits determining the body plan of planktonic filter feeders. American Naturalist, 194(2). https://doi.org/10.1086/703656

\section{General rights}

Copyright and moral rights for the publications made accessible in the public portal are retained by the authors and/or other copyright owners and it is a condition of accessing publications that users recognise and abide by the legal requirements associated with these rights.

- Users may download and print one copy of any publication from the public portal for the purpose of private study or research.

- You may not further distribute the material or use it for any profit-making activity or commercial gain

- You may freely distribute the URL identifying the publication in the public portal 


\title{
Dense Dwarfs versus Gelatinous Giants: The Trade-Offs and Physiological Limits Determining the Body Plan of Planktonic Filter Feeders
}

\author{
Julia Dölger, ${ }^{1}$ Thomas Kiørboe, ${ }^{2}$ and Anders Andersen ${ }^{1, \star}$ \\ 1. Department of Physics and Centre for Ocean Life, Technical University of Denmark, DK-2800 Kongens Lyngby, Denmark; 2. National \\ Institute of Aquatic Resources and Centre for Ocean Life, Technical University of Denmark, DK-2800 Kongens Lyngby, Denmark
}

Submitted June 8, 2018; Accepted January 11, 2019; Electronically published June 11, 2019

Dryad data: https://dx.doi.org/10.5061/dryad.n8f8gb0.

\begin{abstract}
AвSTRACT: Most marine plankton have a high energy (carbon) density, but some are gelatinous with approximately 100 times more watery bodies. How do those distinctly different body plans emerge, and what are the trade-offs? We address this question by modeling the energy budget of planktonic filter feeders across life-forms, from micronsized unicellular microbes such as choanoflagellates to centimeter-sized gelatinous tunicates such as salps. We find two equally successful strategies, one being small with high energy density (dense dwarf) and the other being large with low energy density (gelatinous giant). The constraint that forces large - but not small- filter feeders to be gelatinous is identified as a lower limit to the size-specific filter area, below which the energy costs lead to starvation. A further limit is found from the maximum size-specific motor force that restricts the access to optimum strategies. The quantified constraints are discussed in the context of other resource-acquisition strategies. We argue that interception feeding strategies can be accessed by large organisms only if they are gelatinous. On the other hand, organisms that use remote prey sensing do not need to be gelatinous, even if they are large.
\end{abstract}

Keywords: gelatinous zooplankton, filter feeding, low Reynolds number fluid dynamics, salps, choanoflagellates.

\section{Introduction}

Gelatinous organisms such as salps, jellyfish, and comb jellies are characterized by a watery body with an energy (carbon) density that is approximately 100 times lower than that of nongelatinous (dense) forms (Kiørboe 2013; Lucas and Dawson 2014). Such organisms have become increasingly recognized for their important roles in the oceanic food web (Alldredge and Madin 1982; Harbison 1992; Bone 1998;

\footnotetext{
* Corresponding author; email: aanders@fysik.dtu.dk. ORCIDs: Dölger, https://orcid.org/0000-0003-4667-4716; Kiørboe, https:// orcid.org/0000-0002-3265-336X; Andersen, https://orcid.org/0000-0002-3831-1707.

Am. Nat. 2019. Vol. 194, pp. E000-E000. (C) 2019 by The University of Chicago. 0003-0147/2019/19402-58534\$15.00. All rights reserved.

DOI: $10.1086 / 703656$
}

Arai 2001; Condon et al. 2012; Henschke et al. 2016), and it has been argued that the watery body makes them capable of surviving in dilute environments due to their large prey encounter surface (Harbison 1992; Acuna 2001; Acuna et al. 2011). Zooplankton body plans do not show only a dichotomy between gelatinous and dense organisms with few intermediate densities (Kiørboe 2013), but displayed in the trait space of energy content $E$ and energy density $\rho$, three distinct groups are notable (fig. 1): ${ }^{1}$ protozoans with small energy content and "natural" dense body composition, gelatinous organisms (i.e., pelagic tunicates and jellyfish with large energy content and low energy density), and other zooplankton with large energy content and natural dense body composition. In this study, we aim to rationalize these observations.

Many plankton, including gelatinous organisms, are interception feeders that create a feeding flow past themselves and directly intercept prey rather than use remote prey sensing. The strategy is widespread, but it is demanding since such organisms need to process huge amounts of water to survive in the nutritionally dilute oceanic environment (Kiørboe 2011). Interception feeding involves a fundamental tradeoff: the flow is crucial to collect prey, but energy is required to create the flow. Filter feeders are a special group of interception feeders that pass the feeding flow through fibrous structures where prey is retained. For planktonic filter feeders, here we explore optimal and limiting strategies and the conditions under which dense and gelatinous body plans emerge.

The main components of a filter feeder are the body, the filter, and the motor that drives the feeding flow. The biological motor is made of either flagella, cilia, or muscles. The two dominant groups of planktonic filter feeders that

1. Data underlying figs. 1 and 3-5 and tables 2 and 3 are deposited in the Dryad Digital Repository: https://dx.doi.org/10.5061/dryad.n8f8gb0 (Dölger et al. 2019). 


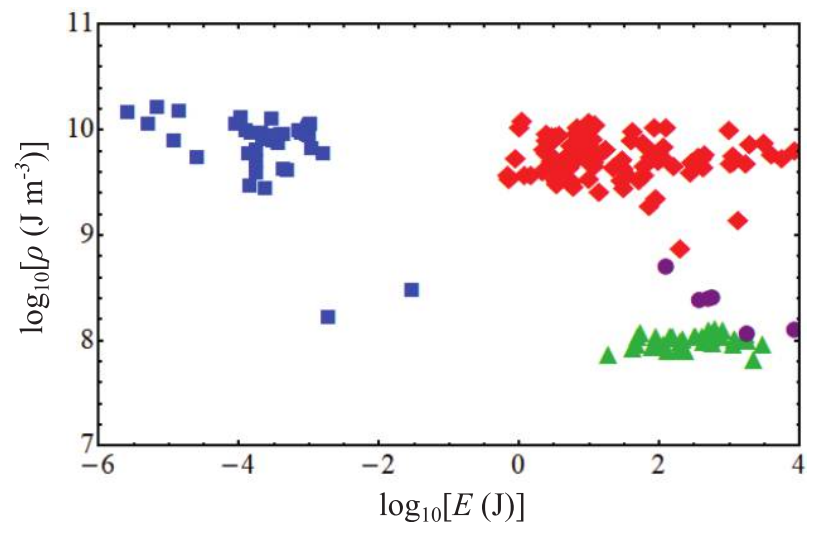

Figure 1: Energy density $\rho$ versus energy content $E$ for zooplankton. Collected data for protozoans (blue squares), tunicates (green triangles), jellyfish (purple circles), and other zooplankton that are mainly copepods (red diamonds) (Kiørboe 2013; Dölger et al. 2019).

we investigate here as examples are choanoflagellates and salps (fig. 2). They are on contrasting ends of the planktonic size spectrum. Choanoflagellates are unicellular and use a sin- gle flagellum to create a feeding current through a collarshaped filter made of strands (microvilli) that extend from the cell (Leadbeater 2015). Salps are gelatinous pelagic tunicates that are up to several centimeters long and use muscle rings in a barrel-shaped body to drive flow through a filter made of submicron-thick mucus strands (Bone 1998). The filter spacing varies surprisingly little from choanoflagellates to salps, and they thus compete for a common source of micron-sized prey in the plankton (Hansen et al. 1994; Sutherland et al. 2010; Lombard et al. 2011; Nielsen et al. 2017). We use these overarching similarities across life-forms to set up a general energetics model of planktonic filter feeders to quantify trade-offs and physiological limits.

Energetics models have been developed by Acuna and collaborators to explore the scope for growth of gelatinous species such as salps (filter feeders) and jellyfish (cruising feeders). Acuna (2001) estimated the filter flow speed for salps that maximizes growth, while Acuna et al. (2011) estimated the cruising speed for jellyfish that optimizes survival at low food concentrations.

We build on the models by Acuna and collaborators, but in our energetics model we use size-specific quantities

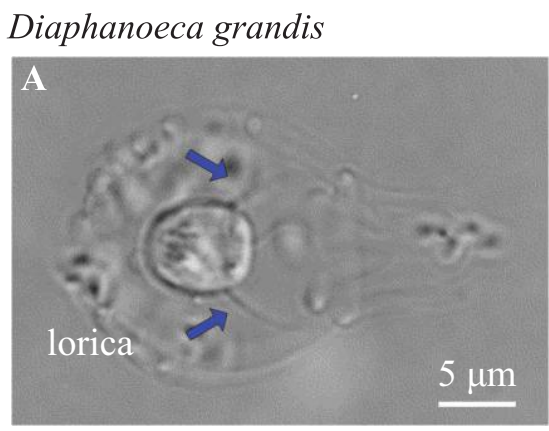

\section{Pegea confoederata}
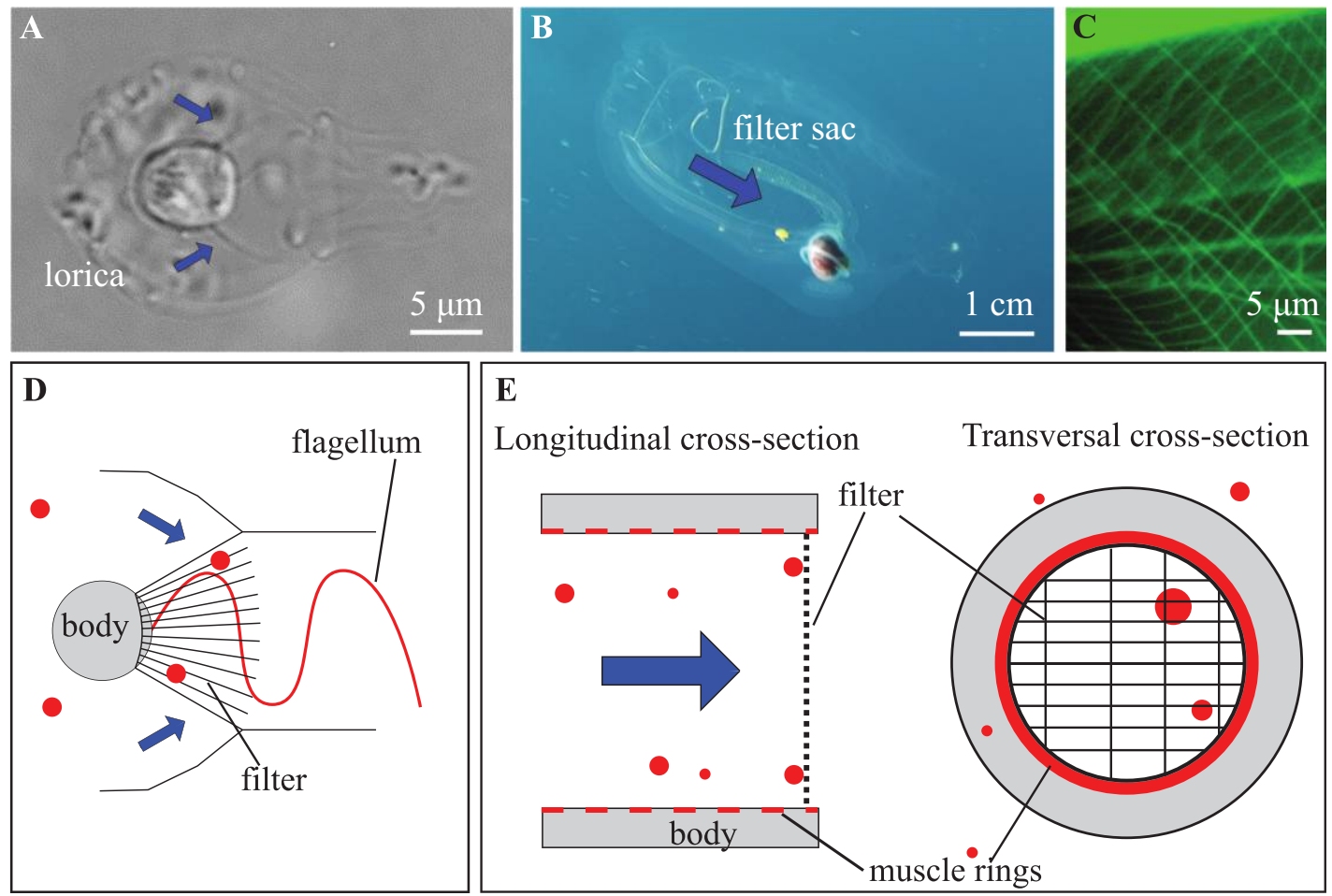

$\mathbf{E}$

Longitudinal cross-section

Transversal cross-section

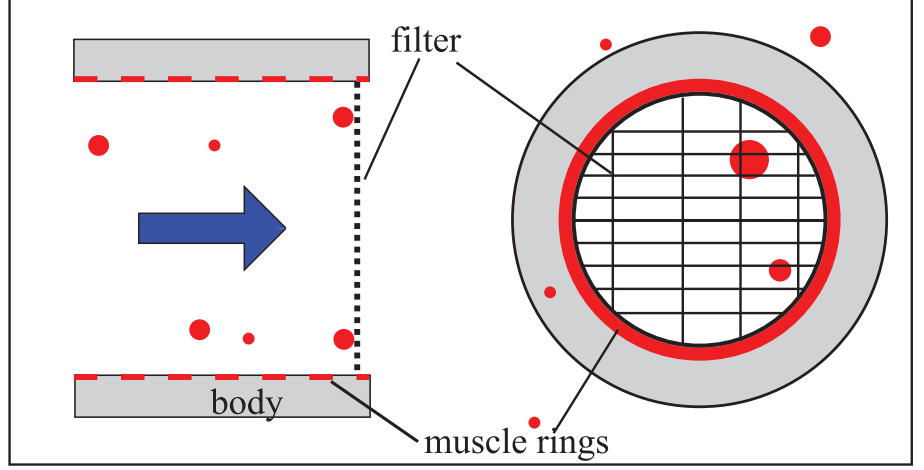

Figure 2: Morphologies and models of planktonic filter feeders. A, Choanoflagellate of species Diaphanoeca grandis with cell, collar-shaped filter, flagellum, and basketlike lorica structure with particles stuck on it. Image courtesy of Lasse Tor Nielsen. B, Salp of species Pegea confoederata with gelatinous barrel-shaped body and filter sac. C, Mucous filter of P. confoederata. Images courtesy of Kelly R. Sutherland. $D, E$, Simplified schematic models of choanoflagellate $(D)$ and salp $(E)$. Red disks and blue arrows indicate prey particles and filter flow directions, respectively. 
that allow us to model planktonic filter feeders across size classes. We show that small organisms can have dense body plans, while larger organisms must either be gelatinous to ensure a sufficiently large filter area or be able to remotely sense prey to survive in the pelagic realm of the ocean. We further demonstrate that motor performance constrains optimality of filter flow speed and body plan.

\section{Energetics Model of Planktonic Filter Feeders}

The scope for growth of an individual - that is, the total energy per time available for growth and reproductionis the energy gain (prey ingestion rate) minus the energy cost (respiration rate). By dividing the scope for growth and other quantities by the energy content of the organism we arrive at energy-specific quantities, which allows us to make comparisons across size classes. The energy content of an organism is assumed to be proportional to its carbon mass (Acuna 2001; Schmidt-Nielsen 2007). The energyspecific scope for growth $H$ is

$$
H=G-R_{\mathrm{f}}-R_{\mathrm{b}},
$$

where $G$ is the energy-specific prey ingestion rate. A glossary of symbols is provided in table 1 . The energy-specific respiration rate is divided into a dynamic part $R_{\mathrm{f}}$ for filter flow creation and a basal part $R_{\mathrm{b}}$ for maintenance. Both $G$ and $R_{\mathrm{f}}$ depend on the filter flow speed $u$, and we can write

$$
H=A u c-k A u^{2}-R_{\mathrm{b}}
$$

where $A$ is the energy-specific filter area, $c$ is the prey concentration in energy per volume, and $k$ is the filter resis- tance that we model as a function of mesh spacing $l$ and strand radius $a$ of the filter (table 2). The parameters $A$, $u, k$, and $R_{\mathrm{b}}$ are traits of the organism, while $c$ is an external parameter that defines the environment. For simplicity we assume that the assimilation efficiency (i.e., the assimilated fraction of the ingested energy) is $100 \%$ and that the energyspecific clearance rate, $Q$, is independent of prey concentration and can be expressed as $Q=A u$. The latter assumption is realistic for filter feeders that capture prey at low concentration (Lehman 1976; Jeschke et al. 2004; Wirtz 2012).

We estimate the dynamic part of the energy-specific respiration rate, $R_{\mathrm{f}}$, as the energy-specific motor force $F=k A u$ times the filter flow speed $u$, thus obtaining the energy-specific power that goes into flow creation (Vogel 1994; Acuna 2001). For simplicity, energy conversion efficiencies are assumed to be $100 \%$. The filter resistance $k$ varies little across life-forms, since observations show that mesh spacing $l$ and strand radius $a$ are similar in different planktonic filter feeders (Bone et al. 1991; Leadbeater 2015). We model $k$ as the low Reynolds number resistance to water flow with viscosity $\mu$ through a filter consisting of parallel cylinders (table 2; Tamada and Fujikawa 1957; Ayaz and Pedley 1999; Nielsen et al. 2017). We assume that the filter will prevent flow circumvention (e.g., by a surrounding channel structure). The additional resistance due to surrounding walls and channel ends is neglected, which is reasonable for fine filters inside a wide cavity, where the resistance due to the filter dominates.

The basal respiration rate is assumed to be proportional to the energy content. This is different from the typical Kleiber-type allometric scaling for which the specific met-

Table 1: Glossary of symbols

\begin{tabular}{lll}
\hline Symbol & \multicolumn{1}{c}{ Description } & \multicolumn{1}{c}{ Unit } \\
\hline$a$ & Filter strand radius & $\mathrm{m}$ \\
$A$ & Energy-specific filter area & $\mathrm{m}^{2} \mathrm{~J}^{-1}$ \\
$c$ & Prey concentration & $\mathrm{J} \mathrm{m}^{-3}$ \\
$D$ & Drag parameter for cruise feeder & $\mathrm{kg} \mathrm{m}^{-3}$ \\
$E$ & Energy content of organism & $\mathrm{J}$ \\
$F$ & Energy-specific motor force & $\mathrm{N} \mathrm{J}^{-1}$ \\
$G$ & Energy-specific prey ingestion rate & $\mathrm{s}^{-1}$ \\
$H$ & Energy-specific scope for growth & $\mathrm{s}^{-1}$ \\
$k$ & Filter resistance & $\mathrm{kg} \mathrm{m}^{-2} \mathrm{~s}^{-1}$ \\
$l$ & Filter mesh spacing & $\mathrm{m}$ \\
$Q$ & Energy-specific clearance rate & $\mathrm{m}^{3} \mathrm{~J}^{-1} \mathrm{~s}^{-1}$ \\
$R_{\mathrm{b}}$ & Energy-specific basal respiration rate & $\mathrm{s}^{-1}$ \\
$R_{\mathrm{f}}$ & Energy-specific dynamical respiration rate & $\mathrm{s}^{-1}$ \\
$R_{\max }$ & Maximum energy-specific dynamical respiration rate & $\mathrm{s}^{-1}$ \\
$S$ & Energy-specific projected body area of cruise feeder & $\mathrm{m}^{2} \mathrm{~J}^{-1}$ \\
$u$ & Filter flow speed & $\mathrm{m} \mathrm{s}^{-1}$ \\
$v$ & Swimming speed of cruise feeder & $\mathrm{m} \mathrm{s}^{-1}$ \\
$V$ & Body volume of organism & $\mathrm{m}^{3}$ \\
$\mu$ & Viscosity of water & $\mathrm{Pa} \mathrm{s}^{-3}$ \\
$\rho$ & Energy density of organism & $\mathrm{J} \mathrm{m}^{-3}$ \\
\hline
\end{tabular}


Table 2: Parameter values for Diaphanoeca grandis and Pegea confoederata

\begin{tabular}{llcc}
\hline Parameter & \multicolumn{1}{c}{ Description } & D. grandis & P. confoederata \\
\hline$u\left(\mathrm{~m} \mathrm{~s}^{-1}\right)$ & Filter flow speed & $7 \cdot 10^{-6}$ & $2 \cdot 10^{-3}$ \\
$l(\mathrm{~m})$ & Filter mesh spacing & $5 \cdot 10^{-7}$ & $2 \cdot 10^{-6}$ \\
$a(\mathrm{~m})$ & Filter strand radius & $8 \cdot 10^{-8}$ & $5 \cdot 10^{-8}$ \\
$k\left(\mathrm{~kg} \mathrm{~m}^{-2} \mathrm{~s}^{-1}\right)$ & Filter resistance & $3 \cdot 10^{4}$ & $3 \cdot 10^{3}$ \\
$R_{\mathrm{b}}\left(\mathrm{s}^{-1}\right)$ & Energy-specific basal respiration rate & $1 \cdot 10^{-6}$ & $1 \cdot 10^{-6}$ \\
$A\left(\mathrm{~m}^{2} \mathrm{~J}^{-1}\right)$ & Energy-specific filter area & $3 \cdot 10^{-4}$ & $6 \cdot 10^{-6}$ \\
\hline
\end{tabular}

Note: Flow speed, mesh spacing, strand radius, and area of the filter were taken from observed values on the respective species (Bone et al. 1991; Sutherland et al. 2010; Nielsen et al. 2017). For A, the filter area was divided by the energy content. The energy content of $P$. confoederata was calculated from its observed carbon mass and with the conversion factor $5.5 \cdot 10^{7} \mathrm{~J} \mathrm{kgC}^{-1}$ (Acuna 2001), while for D. grandis it was calculated from the cell volume times the "natural" energy density $\rho_{\text {nat }}=1 \cdot 10^{10} \mathrm{~J} \mathrm{~m}^{-3}$ (Kiørboe 2013; Nielsen et al. 2017). The respiration rates were converted from oxygen to energy consumption per time with the conversion factor $20 \mathrm{JmLO}_{2}^{-1}$ (Schmidt-Nielsen 2007). The filter resistance coefficient was calculated as $k=(8 \pi \mu / l) /\left(1-2 \ln \tau+\tau^{2} / 6-\tau^{4} / 144\right)$, where $\tau=2 \pi a / l$ and $\mu=1 \cdot 10^{-3} \mathrm{Pas}$ is the viscosity of water (Tamada and Fujikawa 1957). Data used for parameter value calculations are deposited in the Dryad Digital Repository: https://dx.doi.org/10.5061/dryad.n8f8gb0 (Dölger et al. 2019).

abolic rate decreases with increasing size. Such nonlinear scaling applies within individual groups of planktonic organisms (e.g., protozoans and tunicates), but it is empirically well established that the total respiration rate is proportional to carbon content across groups (Makarieva et al. 2008; Kiørboe and Hirst 2014). Thus, we have a constant energyspecific basal respiration rate, $R_{\mathrm{b}}$. Only total respiration rates are usually recorded in experiments. The measured total respiration rates are used here as estimates for $R_{\mathrm{b}}$, since the dynamic respiration rate, $R_{\mathrm{f}}$, is variable and found to be well below $10 \%$ of the total respiration rate (Crawford 1992; Svetlichny and Hubareva 2005). In the following, we assume that $R_{\mathrm{f}}$ is smaller or maximally equal to $R_{\mathrm{b}}$.

Evidently, our model of planktonic filter feeders does not apply to all organisms that are typically classified as filter feeders. First of all, the model applies only to filter feeders that create low Reynolds number flows dominated by viscous friction. Further, the flow-creating motor is assumed to be separate from the filter, unlike in ciliated filters where pump elements on the filter strands themselves create an effective slip velocity, such as in bivalves, brachiopods, or bryozoans (Riisgård and Larsen 2010). Most of those ciliary suspension feeders, however, live in the benthic zone where more prey are available, while we consider pelagic plankton that live in a very dilute environment where prey concentration is more limiting.

\section{Characteristics of Filter Feeding}

We first explore how the prey concentration, $c$, the energyspecific filter area, $A$, and the filter flow speed, $u$, affect the energy-specific scope for growth, and we determine favorable trait combinations. An optimal strategy in a stable environment is to maximize the energy-specific scope for growth (Acuna 2001). The filter flow speed that maximizes the energy-specific scope for growth $H$ in equation (2) with the other parameters fixed is

$$
u_{H}=\frac{c}{2 k},
$$

and with this strategy we obtain the maximum energyspecific scope for growth as

$$
H_{\max }=\frac{A c^{2}}{4 k}-R_{\mathrm{b}} .
$$

Thus, to achieve a high $H_{\max }$, the energy-specific filter area, $A$, should be large.

An alternative optimization strategy, which is especially valuable in a fluctuating environment with competitors for food, is to be able to survive at low prey concentrations (Tilman 1982; Acuna et al. 2011). The limiting concentration below which the organism cannot sustain itself follows from setting $H$ equal to zero in equation (2):

$$
c_{H=0}=k u+\frac{R_{\mathrm{b}}}{A u} .
$$

Minimization of the function (5) with respect to $u$ results in the filter flow speed

$$
u_{\mathrm{c}}=\sqrt{\frac{R_{\mathrm{b}}}{k A}}
$$

and leads to the minimum limiting concentration

$$
c_{\min }=2 \sqrt{\frac{k R_{\mathrm{b}}}{A}},
$$

which decreases with $A$. Thus, a high energy-specific filter area also favors this strategy.

To test the model, we compare the observed filter flow speeds of the choanoflagellate Diaphanoeca grandis and the salp Pegea confoederata at different prey concentrations 
with the two optimum strategies (fig. 3). We use observed and estimated parameter values for $A, k$, and $R_{\mathrm{b}}$ (table 2 ). The contour line $H=0$ divides feasible and unfeasible strategies, and it is given by the limiting concentration $c_{H}={ }_{0}$ defined in equation (5). For low speeds where the basal respiration dominates, it decreases with $c_{H}={ }_{0} \approx R_{\mathrm{b}} /(A u)$, while at high speeds, where the filter resistance dominates, it increases as $c_{H}={ }_{0} \approx k u$. The energy-specific filter area is larger for $D$. grandis than for $P$. confoederata, whereas the opposite holds for the filter flow speeds (table 2). The observed speeds $u$ in both organisms are substantially lower than the optimum speeds of equation (3) for maximization of the growth rate at typical concentrations $c_{\text {low }}$ and $c_{\text {mean }}$ (tables 2,3 ). The observed low speeds can maximize $H$ at very low concentrations (Acuna 2001), but the strategy is unfeasible because there $H_{\max }$ is negative (fig. 3). The observed speed in $D$. grandis can also not be explained by the optimum (6) for survival at low prey concentrations (fig. $3 A$ ), whereas the observed speed in $P$. confoederata is near the optimum value $u_{c}$ (fig. $3 B$ ). This result supports the suggestion that gelatinous filter feeders are capable of surviving at low prey concentrations (Harbison 1992; Acuna 2001).

The energy-specific respiration rate $R_{\mathrm{b}}$ is roughly constant across life-forms, as discussed, and the filter resistance $k$ also varies only one order of magnitude within the variation in body length of four orders of magnitude (fig. 2; table 2). To explore the model predictions further, we now consider a "standard" filter feeder with constant $R_{\mathrm{b}}$ and $k$, for which we use mean values (table 3 ). With this simplifying assumption, we are left with three main variables: $c, A$, and $u$. The prey concentration $c$ is determined by the environment (table 3 ), whereas $A$ and $u$ define morphological and behavioral key traits of the filter feeder (fig. 4A). Here the contour line $H=0$, which again separates feasible and unfeasible strategies, is characterized by the curve

$$
A_{H=0}=\frac{R_{\mathrm{b}}}{c u-k u^{2}} .
$$

At low speeds, we find the decreasing function $A_{H=0} \approx R_{\mathrm{b}} /(\mathrm{cu})$, and $A_{H}=0$ goes to infinity when $u$ approaches $c / k$. The maximum speed $c / k$, above which we have $G-R_{\mathrm{f}}<0$ and thus negative $H$, defines a general limit. However, most observed filter feeders are far from reaching this maximum speed, and only a small part of the trait space with positive $H$ is occupied (fig. $4 A$ ). The shown trait combinations are estimated from measured filter flow speeds, filter areas, body sizes, and clearance rates (Alldredge and Madin 1982; Nielsen et al. 2017; table 2). The populated part of the trait space corresponds to a narrow range in energy-specific clearance rate $Q=A u$ (mean \pm SD within purple lines; Alldredge and Madin 1982; Kiørboe and Hirst 2014). This trend of an approximately constant energy-specific clearance rate cannot be explained by the global optimum strategies. However, motor performance may constrain the accessible trait space and provide a possible explanation of the trend, as we demonstrate in the following section.

In summary, in this section we have determined feasible and unfeasible trait combinations, both for our two study organisms D. grandis and P. confoederata and for standard filter feeders that represent filter feeders across planktonic
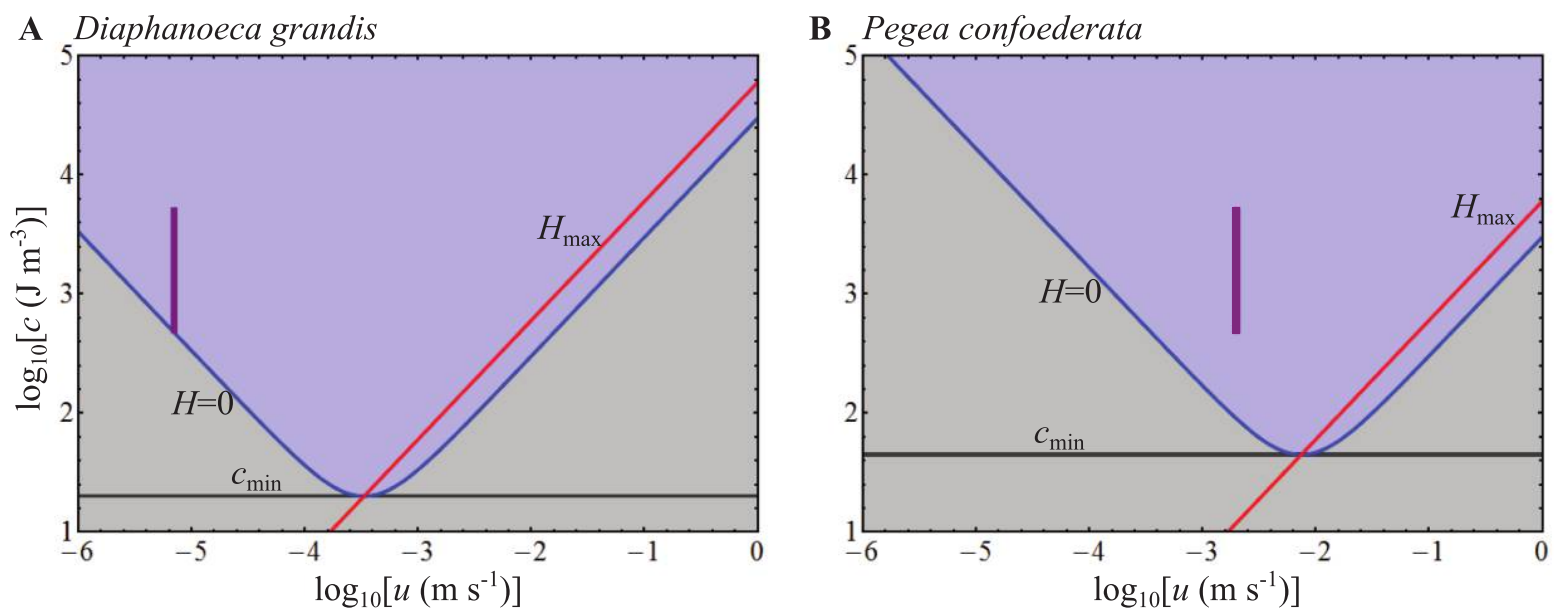

Figure 3: Parameter space defined by the filter flow speed $u$ and the prey concentration $c$ for the choanoflagellate Diaphanoeca grandis (A) and the salp Pegea confoederata $(B)$. The blue line corresponds to the zero scope for growth contour line as calculated from equation (5) and using structural and functional parameters from table 2 (Dölger et al. 2019). This line separates regions with positive (blue shading) and negative (gray shading) scope for growth. The filter flow speeds that yield maximum scope for growth $H_{\max }$ at given prey concentrations are shown by the red line (eq. [3]). The lowest prey concentration $c_{\min }$ at which the organism can survive is shown as the black line (eq. [7]). The observed filter flow speed (table 2) and a typical range of prey concentrations between $c_{\text {low }}$ and $c_{\text {mean }}$ (table 3 ) are shown by a purple bar. 
Table 3: Parameter values and ranges for the "standard" planktonic filter feeders

\begin{tabular}{llc}
\hline Parameter & \multicolumn{1}{c}{ Description } & Value/range \\
\hline$c_{\text {low }}\left(\mathrm{J} \mathrm{m}^{-3}\right)$ & Low prey concentration & $5 \cdot 10^{2}$ \\
$c_{\text {mean }}\left(\mathrm{J} \mathrm{m}^{-3}\right)$ & Mean prey concentration & $5 \cdot 10^{3}$ \\
$l(\mathrm{~m})$ & Filter mesh spacing & $1 \cdot 10^{-6}$ \\
$a(\mathrm{~m})$ & Filter strand radius & $1 \cdot 10^{-7}$ \\
$k\left(\mathrm{~kg} \mathrm{~m}^{-2} \mathrm{~s}^{-1}\right)$ & Filter resistance & $1 \cdot 10^{4}$ \\
$R_{\mathrm{b}}\left(\mathrm{s}^{-1}\right)$ & Energy-specific basal respiration rate & $1 \cdot 10^{-6}$ \\
$\log _{10}\left(\mathrm{Q} /\left[\mathrm{m}^{3} \mathrm{~J}^{-1} \mathrm{~s}^{-1}\right]\right)$ & Energy-specific clearance rate & $-8.3 \pm .4$ \\
$R_{\max }\left(\mathrm{s}^{-1}\right)$ & Maximum energy-specific power & $1 \cdot 10^{-6}$ \\
$F_{\max }\left(\mathrm{N} \mathrm{J}^{-1}\right)$ & Maximum energy-specific force & $1 \cdot 10^{-4}$ \\
\hline
\end{tabular}

Note: Observed carbon concentrations based on the abundance of particles with diameter $>2 \mu \mathrm{m}$ were used to calculate energy concentrations with the conversion factor $5.5 \cdot 10^{7} \mathrm{~J} \mathrm{kgC}^{-1}$ (Buck et al. 1996; Acuna 2001). The parameter $c_{\text {low }}$ is estimated as the mean concentration found in regions with the lowest concentrations, and $c_{\text {mean }}$ is estimated as the mean found in regions with the highest concentrations; both are measured in the North Atlantic (Buck et al. 1996). The energy-specific basal respiration rate (and the maximum energy-specific power) were estimated from average measured carbon-specific rates (Kiørboe and Hirst 2014). The maximum energy-specific force was estimated from the highest energy-specific clearance rate in filter feeders using $F_{\max }=k Q_{\max }$. Mean and standard deviation of the energy-specific clearance rates $Q$ were calculated assuming a lognormal distribution with observations from different filter feeders (Alldredge and Madin 1982; Nielsen et al. 2017). Data used for parameter value calculations are deposited in the Dryad Digital Repository: https://dx.doi.org/10.5061/dryad.n8f8gb0 (Dölger et al. 2019).

life-forms. In doing so we have identified the energy-specific filter area and the filter flow speed as the key traits for filter feeders.

\section{Limitations to Body Plan and Filter Flow Speed}

There are, of course, limits to the power and force of the motor that creates the filter flow, and these limits depend on the body plan. One natural constraint is found by assuming that

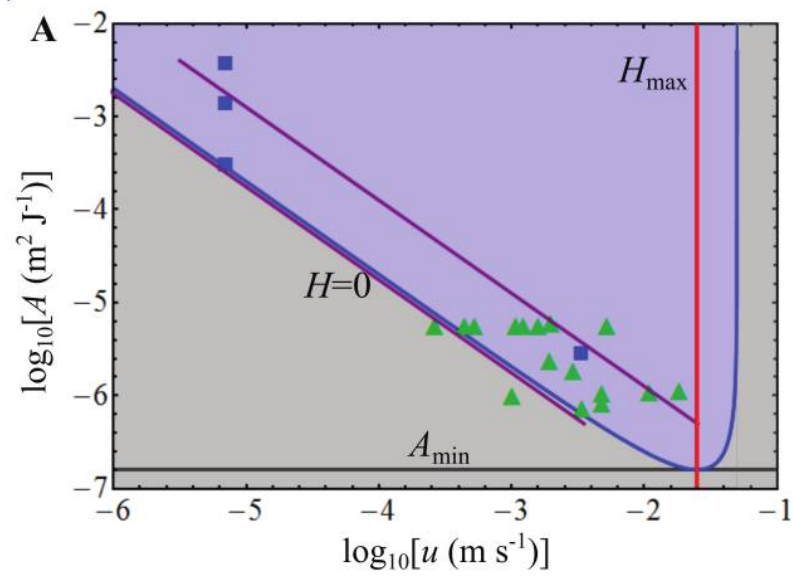

the maximum motor power is proportional to the energy content of the organism. Such proportionality is suggested by several studies on metabolic rates at high activity (Weibel and Hoppeler 2005; Glazier 2014; Meyer-Vernet and Rospars 2016). From this we obtain a constant maximum limit $R_{\max }$ to the energy-specific dynamic respiration rate $R_{\mathrm{f}}$, as has also been found for the energy-specific total respiration rate (Kiørboe and Hirst 2014). We make the simplifying assumption that the maximum motor power is equal to the basal respiration rate (i.e., in the extreme case, the total consumption

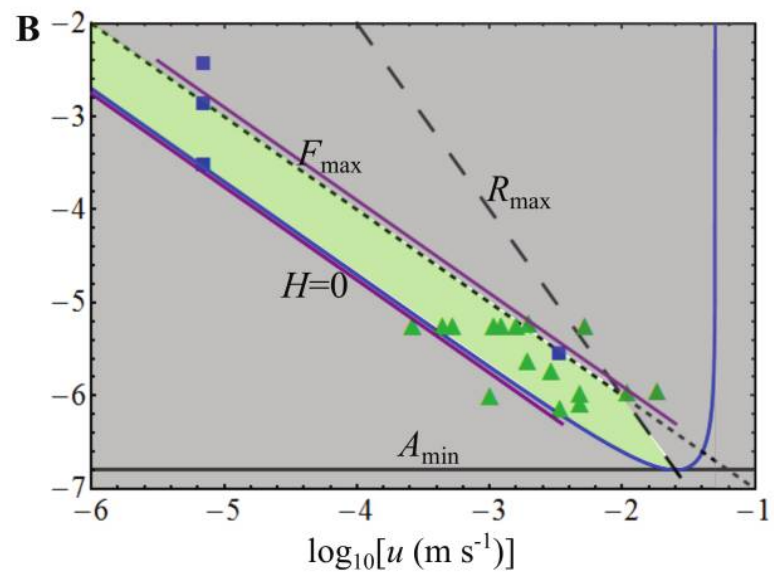

Figure 4: Optima and constraints in the trait space defined by filter flow speed $u$ and energy-specific filter area $A$ for "standard" planktonic filter feeders at a typical low prey concentration $c_{\text {low }}$ (table 2). The blue line corresponds to the zero scope for growth contour line as calculated from equation (8) and using structural and functional parameters from table 2. This line separates regions with positive (blue shading in $A$ ) and negative (gray shading) scope for growth. The purple lines reflect the range of clearance rates (log mean $\pm S D$ ) and envelope observed trait combinations (green triangles: tunicates; blue squares: protozoans; Dölger et al. 2019). Also represented are the line for maximum scope for growth $\left(H_{\max } ;\right.$ eq. [3]; $\left.A\right)$ and the lines for a maximum power constraint $\left(R_{\max }\right.$; eq. [9];B) and maximum force constraint $\left(F_{\max } ;\right.$ eq. $\left.[11] ; B\right)$, which define a narrow feasible trait region (green shading) from an unfeasible region above the maximum limits (gray shading). A comparison of observed and modeled feasible trait combinations suggests that planktonic filter feeders are dominantly limited by the maximum force constraint. 
is divided equally between filter flow creation and maintenance). Thus, we have

$$
k A u^{2}<R_{\max }=R_{\mathrm{b}} .
$$

The larger the energy-specific filter area, $A$, the lower the filter flow speed, $u$, that the motor can generate. With the power limit $R_{\max }$, the speed is limited by that maximum

$$
u_{\mathrm{R}}=\sqrt{\frac{R_{\mathrm{max}}}{k A}},
$$

which, with $R_{\max }=R_{\mathrm{b}}$, coincides with $u_{\mathrm{c}}$ in equation (6). A second natural constraint is that of a maximum motor force. Marden and Allen (2002) have found that the maximum force for cyclical motors is proportional to the motor mass. In our model, we can define such a limit as a constant maximum energy-specific force $F_{\max }$ from which we obtain the constraint

$$
k A u<F_{\max } .
$$

Due to the force limit $F_{\max }$, the filter flow speed is limited by the maximum

$$
u_{\mathrm{F}}=\frac{F_{\max }}{k A} .
$$

If both limits are valid as general constraints, the limit to motor performance is ultimately determined by the lower of the two (fig. $4 B$ ).

The feasible combinations of $A$ and $u$ are restricted by the contour line $H=0$ (eq. [8]) and the motor performance limits $R_{\max }$ (eq. [10]) and $F_{\max }$ (eq. [12]). We observe that filter feeders are force limited rather than power limited at typical low prey concentrations (table 3 ; fig. $4 B$ ). This observation can be understood since most filter feeders have an approximately constant energy-specific clearance rate $Q$, as discussed earlier, and using the relation $F=k Q$, this leads to an approximately constant $F$. Further, from the maximum clearance rate, $Q_{\max }$, we estimate the value of the force limit $F_{\text {max }}=k Q_{\text {max }}=1 \cdot 10^{-4} \mathrm{~N} \mathrm{~J}^{-1}$ (table 3).

With all considered constraints, we can calculate an energyspecific filter area, $A_{\min }$, below which $H$ is negative (fig. 4). This is determined as the minimum of the contour line $A_{H=0}$, that is,

$$
A_{\min }=\frac{4 k R_{\mathrm{b}}}{c^{2}} .
$$

The minimum in equation (13) is within the feasible trait space as long as $F_{\max } \geq 2 k R_{\mathrm{b}} / c$ and $R_{\max } \geq R_{\mathrm{b}}$ (see the appendix). In the following section we will show how to determine the maximum energy density from the minimum of the energy-specific filter area.

To summarize this section, we have shown that upper limits on force and power both constrain the accessible trait combinations for filter feeders, and most importantly we have shown that the energy-specific filter area displays a minimum below which filter feeding is unfeasible.

\section{Maximum Energy Density}

As discussed in the introduction, zooplankton separate into three distinct groups in the trait space of energy content $E$ and energy density $\rho$ (i.e., protozoans, gelatinous organisms, and other zooplankton; fig. 1). We now argue that these distinct body plans are a consequence of the feeding strategies. The argument for filter feeders is that there exists a minimum energy-specific filter area, $A_{\min }$, below which filter feeding is unfeasible (eq. [13]; appendix). We expect variability but no size-dependent trends in the details of body shape, and we therefore disregard such effects in our analysis. We assume that the filter area is proportional to the body surface area, and we estimate it as $V^{2 / 3}$, where $V=E / \rho$ is the body volume. With the relation we can determine from $A_{\text {min }}$ a maximum energy density, $\rho_{\max }$ as a function of the energy content, $E$, as

$$
\rho_{\max }=\frac{1}{A_{\min }^{3 / 2} E^{1 / 2}} .
$$

For a typical low prey concentration $c_{\text {low }}=5 \cdot 10^{2} \mathrm{~J} \mathrm{~m}^{-3}$, we find $A_{\min }=2 \cdot 10^{-7} \mathrm{~m}^{2} \mathrm{~J}^{-1}$ and our prediction of the maximum energy density $\rho_{\max }$ (fig. $5 A$ ). The natural energy density of approximately $\rho_{\text {nat }}=1 \cdot 10^{10} \mathrm{~J} \mathrm{~m}^{-3}$ (Kiørboe 2013; Dölger et al. 2019) is feasible for unicellular filter feeders with small energy content. Their small size allows them a sufficiently large energy-specific filter area with no need to be gelatinous (dense dwarfs). However, organisms with large energy content are forced by equation (14) to have a lower energy density to sustain a large enough prey encounter surface (gelatinous giants). The maximum energy density depends not only on the energy content but also on the prey concentration. By combining equations (13) and (14), we obtain the expression

$$
\rho_{\max }=\frac{c^{3}}{8 k^{3 / 2} R_{\mathrm{b}}^{3 / 2} E^{1 / 2}},
$$

which is valid when $F_{\max } \geq 2 k R_{\mathrm{b}} / c$ and $R_{\max } \geq R_{\mathrm{b}}$ (appendix). The formula suggests that $\rho_{\max }$ increases strongly (cubically) with the prey concentration $c$. For protozoans, we find that $\rho_{\text {max }}$ is well above the natural energy density $\rho_{\text {nat }}$ even for prey concentrations below $c_{\text {low }}$, whereas for tunicates the constraint is much more restrictive (fig. $5 B$ ).

\section{Discussion}

In this article, we have laid out an energetics model of filter feeders. When analyzing the model, we have used the fact that the filter resistance, $k$, and the energy-specific respiration rate, $R_{\mathrm{b}}$, are roughly constant across size classes, and we have identified the energy-specific filter area, $A$, and the filter flow speed, $u$, as the key traits that characterize filter 

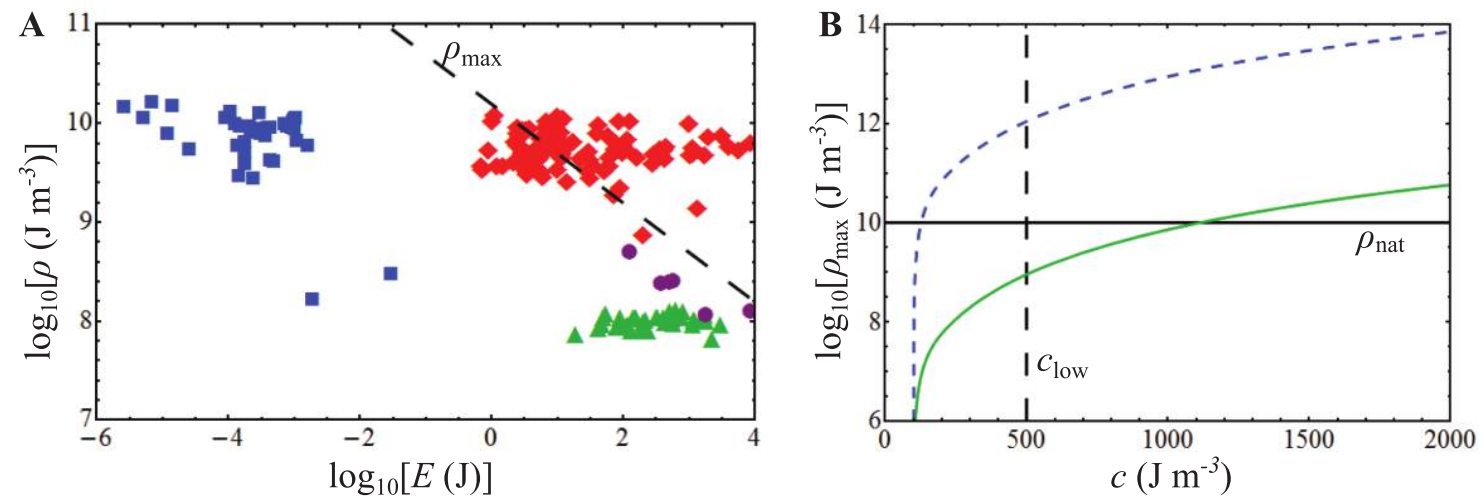

Figure 5: Energy density limits. $A$, Trait space defined by the energy content $E$ and the energy density $\rho$. The collected data for protozoans (blue squares), tunicates (green triangles), jellyfish (purple circles), and other zooplankton that are mainly copepods (red diamonds) are the same as in figure 1 . The black, dashed line indicates the maximum feasible energy density $\rho_{\text {max }}$ for filter feeders at low prey concentration $c_{\text {low }}$ (eq. [14]). $B$, The maximum energy density $\rho_{\max }$ for filter feeders as a function of prey concentration $c$ (eqq. [13], [14], [A14]). Lines are shown for protozoans with low energy content $E=2 \cdot 10^{-4} \mathrm{~J}$ (blue, dashed) and for tunicates with high energy content $E=3 \cdot 10^{2} \mathrm{~J}$ (green, solid). The vertical (black, dashed) line indicates the prey concentration $c_{\text {low }}$ that is used in $A$, and the horizontal (black, solid) line indicates the "natural" energy density $\rho_{\text {nat }}=1 \cdot 10^{10} \mathrm{~J} \mathrm{~m}^{-3}$ found in nongelatinous organisms.

feeders. The interplay in the model between gain and cost of energy has the key implication that a minimum energyspecific filter area is necessary to collect enough food to sustain a living. From this minimum energy-specific filter area, $A_{\text {min }}$, we have identified a maximum sustainable energy density, $\rho_{\max }$, as a function of energy content, $E$, and we have demonstrated a pressure toward being gelatinous that increases with increasing size, thus rationalizing the occurrence of gelatinous body plans only in large plankton.

Observations suggest that zooplankton are either dense or gelatinous, with few intermediate species (fig. 1; Kiørboe 2013). Our proposed scaling of the maximum energy density with energy content would suggest the existence of organisms with intermediate energy densities in the range of energy contents between $10^{-2}$ and $1 \mathrm{~J}$. However, this size range with only a few observations marks the transition between unicellular and multicellular organisms, with the latter typically consisting of 100 or more cells, hence the gap in energy content (fig. 1).

To what extent can our arguments be generalized to other interception feeders that do not possess a filter? For large interception feeders that cruise through the water and directly intercept prey on their body, such as jellyfish, Acuna et al. (2011) developed a model for the scope for growth. For those organisms, the main energy cost was assumed to be due to the drag force on the swimming body. With this assumption, we can write the energy-specific scope for growth for cruise feeders analogous to the filter feeder model (eq. [2]) as

$$
H=S v c-D S v^{3}-R_{\mathrm{b}},
$$

where $S$ is the energy-specific projected body area in the flow direction, $D$ is a constant proportional to the drag coeffi- cient, and $v$ is the swimming speed. The main difference from the filter feeders is contained in the scaling of the flow producing power with $v^{3}$ instead of $v^{2}$ due to the higher Reynolds number of the feeding flow. However, we obtain similar qualitative features of the model, and we can determine a minimum energy-specific area as

$$
S_{\min }=\frac{3^{3 / 2} D^{1 / 2} R_{\mathrm{b}}}{2 c^{3 / 2}}
$$

Below $S_{\min }$, the energy-specific scope for growth is negative. Limits to the body plan follow from equation (14) with $S_{\text {min }}$ instead of $A_{\min }$, and thus our arguments appear to hold for large interception feeders in general (fig. $5 A$ ).

For the group of other zooplankton with large energy content and energy density above $\rho_{\max }$, direct interception feeding in the pelagic zone would not be feasible, since those organisms have too low energy-specific body surface areas (fig. $5 A$ ). Rather than compensating for this by being gelatinous, these organisms have evolved advanced sensing capabilities, such as flow sensing or vision, to perceive their prey individually and from a distance (Martens et al. 2015), thus effectively increasing their encounter surface area beyond their body surface area (Acuna et al. 2011).

So far we have argued for an upper limit to the energy density, but there must also exist a limit to how gelatinous an organism can be. Generally we can argue that at large energyspecific filter areas, $H$ asymptotically approaches its largest value $F_{\max } c / k-R_{\mathrm{b}}$ within the allowed trait combinations (fig. 4B). Most organisms in the observed range of trait combinations are within a high percentage of this largest value, and we speculate that the diminishing return makes further dilution unprofitable, even if the costs are very small. The 
costs associated with being gelatinous are poorly defined. However, in general the need for structures to distribute resources and maintain the integrity of the organism (e.g., in the face of turbulence) increases with water content, as does the cost of swimming because a larger body implies a larger drag (Kiørboe 2013).

We have demonstrated that physiological constraints ultimately limit the motor performance and hence the ability of filter feeders to perform optimally. We now compare the observed energy-specific forces of filter feeders to the constant motor mass-specific forces for cyclical motors found by Marden and Allen (2002). Those authors found a constant motor mass-specific force of $57 \mathrm{~N} \mathrm{~kg}^{-1}$, around which a wide range of motors cluster tightly, including biological motors such as swimming fish. With the measured average energyspecific clearance rate $Q=5 \cdot 10^{-9} \mathrm{~m}^{3} \mathrm{~s}^{-1} \mathrm{~J}^{-1}$ and a typical filter resistance $k=1 \cdot 10^{4} \mathrm{~kg} \mathrm{~m}^{-2} \mathrm{~s}^{-1}$ (table 3), we can calculate the energy-specific forces used to create the feeding current to approximately $F=5 \cdot 10^{-5} \mathrm{~N} \mathrm{~J}^{-1}$ as average for planktonic filter feeders. To convert to force per motor mass, we multiply with the ratio of motor energy density to motor mass density. The motor mass density can be approximated by the density of water $\left(1 \cdot 10^{3} \mathrm{~kg} \mathrm{~m}^{-3}\right)$, and we estimate the motor energy density as $\rho_{\text {nat }}$ (Acuna 2001; Kiørboe 2013). We thus get a motor mass-specific force of around $500 \mathrm{~N} \mathrm{~kg}^{-1}$ for planktonic filter feeders, which is approximately 10 times larger than the universal value found by Marden and Allen (2002). However, small biological motors are underrepresented in their data set. The few very small motors characterized by Marden and Allen, such as swimming bacteria, are not classified in the group of constant motor mass-specific force but exhibit increasing motor mass-specific forces with decreasing size, even exceeding $500 \mathrm{~N} \mathrm{~kg}^{-1}$. The motor massspecific forces produced by copepods during escape jumps (Kiørboe et al. 2010; Svetlichny et al. 2018) are similarly an order of magnitude larger than the universal value proposed by Marden and Allen (2002). This suggests special circumstances for small biological motors, which need to be further explored.

\section{Acknowledgments}

We are thankful to Friederike Prowe and Tomas Bohr for stimulating discussions. This work was supported by the Villum Foundation through the Centre for Ocean Life and by the Independent Research Fund Denmark (grant 701400033B).

\section{APPENDIX}

\section{Minimum Energy-Specific Filter Area}

Here we detail how the minimum energy-specific filter area is calculated in our model. The feasible filter-feeding strat- egies (green region in fig. $4 B$ ) are defined as combinations of energy-specific filter area $A$ and filter flow speed $u$ that fulfill the following conditions.

1. The energy-specific growth rate is nonnegative, that is,

$$
H=A u c-k A u^{2}-R_{\mathrm{b}} \geq 0 .
$$

2. The energy-specific power to create the feeding flow is maximally equal to $R_{\max }$, that is,

$$
k A u^{2} \leq R_{\max } .
$$

3. The energy-specific force to create the feeding flow is maximally equal to $F_{\max }$, that is,

$$
k A u \leq F_{\max } .
$$

The minimum energy-specific filter area is defined as the minimum $A$ within the feasible region, and we shall consider three different cases depending on the values of $R_{\max }$ and $F_{\max }$.

In the first case, which is discussed as the relevant case in the derivation of equation (13), the limit on positive growth (A1) determines the minimum $A$. This case is defined by the conditions

$$
\begin{aligned}
& R_{\max } \geq R_{\mathrm{b}}, \\
& F_{\text {max }} \geq \frac{2 k R_{\mathrm{b}}}{c} ;
\end{aligned}
$$

that is, here the power limit and the force limit are large enough to not constrain $A_{\text {min }}$. We find this lower limit by minimizing $A_{H=0}$ (eq. [8]) with respect to $u$, which leads to

$$
A_{\min }=\frac{4 k R_{\mathrm{b}}}{c^{2}}
$$

at the filter flow speed

$$
u_{\min }=\frac{c}{2 k} .
$$

In the second case, the power limit (A2) dominates, and the conditions defining this case are

$$
\begin{aligned}
& R_{\max } \leq R_{\mathrm{b}}, \\
& F_{\max } \geq \frac{k\left(R_{\max }+R_{\mathrm{b}}\right)}{c} .
\end{aligned}
$$

The minimum $A$ is calculated here as the intersection of the contour line $H=0$ and the contour line $k A u^{2}=R_{\max }$, and it takes the form

$$
A_{\min }=\frac{k\left(R_{\max }+R_{\mathrm{b}}\right)^{2}}{c^{2} R_{\max }}
$$


at the filter flow speed

$$
u_{\min }=\frac{c}{2 k}\left(1-\frac{R_{\mathrm{b}}-R_{\max }}{R_{\mathrm{b}}+R_{\max }}\right) .
$$

In the third case, the force limit (A3) dominates, and the conditions defining this case are

$$
\begin{aligned}
R_{\max } & \geq \frac{F_{\max } c}{k}-R_{\mathrm{b}}, \\
\frac{k R_{\mathrm{b}}}{c} & \leq F_{\max } \leq \frac{2 k R_{\mathrm{b}}}{c} .
\end{aligned}
$$

The minimum $A$ is calculated in this case as the intersection of the contour line $H=0$ and the contour line $k A u=F_{\max }$, and it takes the form

$$
A_{\min }=\frac{F_{\max }^{2}}{F_{\max } c-k R_{\mathrm{b}}}
$$

at the filter flow speed

$$
u_{\min }=\frac{c}{2 k}\left(1-\frac{2 k R_{\mathrm{b}}-F_{\max } c}{F_{\max } c}\right) .
$$

Having determined $A_{\min }$ in the three cases, it is possible to use equation (14) to obtain the corresponding expressions for the maximum energy density as a function of the energy content.

\section{Literature Cited}

Acuna, J. L. 2001. Pelagic tunicates: why gelatinous? American Naturalist 158:100-107.

Acuna, J. L., A. Lopez-Urrutia, and S. Colin. 2011. Faking giants: the evolution of high prey clearance rates in jellyfishes. Science 333:16271629.

Alldredge, A. L., and L. P. Madin. 1982. Pelagic tunicates: unique herbivores in the marine plankton. Bioscience 32:655-663.

Arai, M. N. 2001. Pelagic coelenterates and eutrophication: a review. Hydrobiologia 451:69-87.

Ayaz, F., and T. J. Pedley. 1999. Flow through and particle interception by an array of closely-spaced circular cylinders. European Journal of Mechanics B 18:173-196.

Bone, Q. 1998. The biology of pelagic tunicates. Oxford University Press, Oxford.

Bone, Q., J. C. Braconnot, and K. P. Ryan. 1991. On the pharyngeal feeding filter of the salp Pegea confoederata (Tunicata: Thaliacea) Acta Zoologica 72:55-60.

Buck, K. R., F. P. Chavez, and L. Campbell. 1996. Basin-wide distributions of living carbon components and the inverted trophic pyramid of the central gyre of the North Atlantic Ocean. Aquatic Microbial Ecology 10:283-298.

Condon, R. H., W. M. Graham, C. M. Duarte, K. A. Pitt, C. H. Lucas, S. H. D. Haddock, K. R. Sutherland, et al. 2012. Questioning the rise of gelatinous zooplankton in the world's oceans. Bioscience 62:160-169.
Crawford, D. W. 1992. Metabolic cost of motility in planktonic protists: theoretical considerations on size scaling and swimming speed. Microbial Ecology 24:1-10.

Dölger, J., T. Kiørboe, and A. Andersen. 2019. Data from: Dense dwarfs versus gelatinous giants: the trade-offs and physiological limits determining the body plan of planktonic filter feeders. American Naturalist, Dryad Digital Repository, https://dx.doi.org/10.5061 /dryad.n8f8gb0.

Glazier, D. S. 2014. Scaling of metabolic scaling within physical limits. Systems 2:425-450.

Hansen, B., P. K. Bjornsen, and P. J. Hansen. 1994. The size ratio between planktonic predators and their prey. Limnology and Oceanography 39:395-403.

Harbison, G. R. 1992. The gelatinous inhabitants of the ocean interior. Oceanus 35:18-23.

Henschke, N., J. D. Everett, A. J. Richardson, and I. M. Suthers. 2016. Rethinking the role of salps in the ocean. Trends in Ecology and Evolution 31:720-733.

Jeschke, J. M., M. Kopp, and R. Tollrian. 2004. Consumer-food systems: why type I functional responses are exclusive to filter feeders. Biological Reviews 79:337-349.

Kiørboe, T. 2011. How zooplankton feed: mechanisms, traits and trade-offs. Biological Reviews 86:311-339.

- 2013. Zooplankton body composition. Limnology and Oceanography 58:1843-1850.

Kiørboe, T., A. Andersen, V. J. Langlois, and H. H. Jakobsen. 2010. Unsteady motion: escape jumps in planktonic copepods, their kinematics and energetics. Journal of the Roval Societv Interface 7:1591-1602.

Kiørboe, T., and A. G. Hirst. 2014. Shifts in mass scaling of respiration, feeding, and growth rates across life-form transitions in marine pelagic organisms. American Naturalist 183:E119-E130.

Leadbeater, B. S. C. 2015. The choanoflagellates: evolution, biology and ecology. Cambridge University Press, Cambridge.

Lehman, J. T. 1976. The filter-feeder as an optimal forager, and the predicted shapes of feeding curves. Limnology and Oceanography 21:501-516.

Lombard, F., E. Selander, and T. Kiørboe. 2011. Active prey rejection in the filter-feeding appendicularian Oikopleura dioica. Limnology and Oceanography 56:1504-1512.

Lucas, C. H., and M. N. Dawson. 2014. What are jellyfishes and thaliaceans and why do they bloom? Pages 9-44 in Kylie A. Pitt and Cathy H. Lucas, eds. Jellyfish blooms. Springer, Dordrecht.

Makarieva, A. M., V. G. Gorshkov, B.-L. Li, S. L. Chown, P. B. Reich, and V. M. Gavrilov. 2008. Mean mass-specific metabolic rates are strikingly similar across life's major domains: evidence for life's metabolic optimum. Proceedings of the National Academv of Sciences of the USA 105:16994-16999.

Marden, J. H., and L. R. Allen. 2002. Molecules, muscles, and machines: universal performance characteristics of motors. Proceedings of the National Academv of Sciences of the USA 99:4161-4166.

Martens, E. A., N. Wadhwa, N. S. Jacobsen, C. Lindemann, K. H. Andersen, and A. Visser. 2015. Size structures sensory hierarchy in ocean life. Proceedings of the Roval Societv B 282:20151346.

Meyer-Vernet, N., and J. P. Rospars. 2016. Maximum relative speeds of living organisms: why do bacteria perform as fast as ostriches? Physical Biology 13:066006.

Nielsen, L. T., S. S. Asadzadeh, J. Dölger, J. H. Walther, T. Kiørboe, and A. Andersen. 2017. Hydrodynamics of microbial filter feeding. Proceedings of the National Academv of Sciences of the USA 114:9373-9378. 
Riisgård, H. U., and P. S. Larsen. 2010. Particle capture mechanisms in suspension-feeding invertebrates. Marine Ecology Progress Series 418:255-293.

Schmidt-Nielsen, K. 2007. Animal physiology: adaptation and environment. Cambridge University Press, Cambridge.

Sutherland, K. R., L. P. Madin, and R. Stocker. 2010. Filtration of submicrometer particles by pelagic tunicates. Proceedings of the National Academy of Sciences of the USA 107:15129-15134.

Svetlichny, L. S., and E. Hubareva. 2005. The energetics of Calanus euxinus: locomotion, filtration of food and specific dynamic action. Journal of Plankton Research 27:671-682.

Svetlichny, L., P. S. Larsen, and T. Kiørboe. 2018. Swim and fly: escape strategy in neustonic and planktonic copepods. Iournal of Experimental Biology 221:167262.

Tamada, K., and H. Fujikawa. 1957. The steady two-dimensional flow of viscous fluid at low Reynolds numbers passing through an infinite row of equal parallel circular cylinders. Quarterly Journal of Mechanics and Applied Mathematics 10:425-432.

Tilman, D. 1982. Competition for a single resource. Pages 43-60 in Resource competition and community structure. Princeton University Press, Princeton, NJ.

Vogel, S. 1994. Life in moving fluids: the physical biology of flow. Princeton University Press, Princeton, NJ.

Weibel, E. R., and H. Hoppeler. 2005. Exercise-induced maximal metabolic rate scales with muscle aerobic capacity. mental Biology 208:1635-1644.

Wirtz, K. W. 2012. Intermittency in processing explains the diversity and shape of functional grazing responses. Oecologia 169:879894.

Editor: Russell Bonduriansky

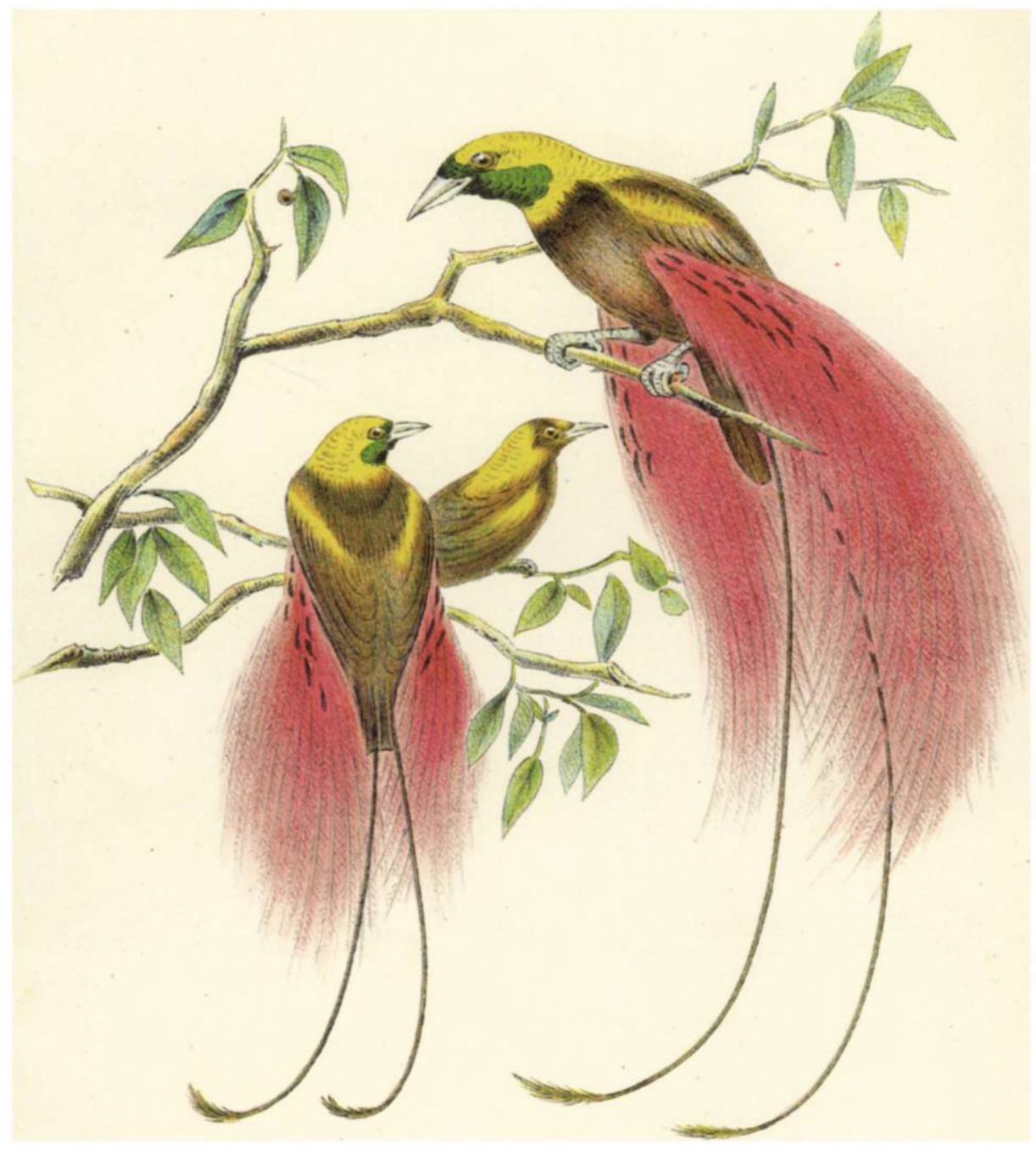

“The Italian naturalist, D’Albertis, for example, encountered several species, notably Paradisea raggiana, along the Fly River-a large stream flowing southeast from the mountains of the interior and emptying into the Gulf of Papua, to the right of Torres Straits. . . The velvety softness of the feathers is as observable in Raggiana as in all birds of paradise, while the exquisite intermingling or suffusion of vivid colors, although at the same time these are quite distinct, is just as inimitable." From "Some Birds of Paradise from New Guinea" by Geo. S. Mead (The American Naturalist, 1894, 28:915-920). 